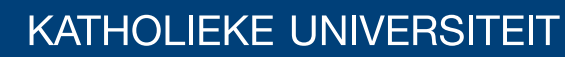 \\ LEUVEN
}

\section{Faculty of Business and Economics}

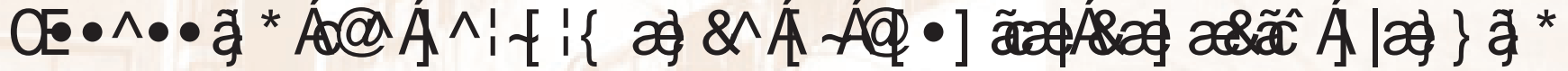
VRRXJKJMP XOURQDQDOVV

* XRI XDQO DIDQG( UN' HP HXOP H HMAN

DEPARTMENT OF DECISION SCIENCES AND INFORMATION MANAGEMENT (KBI) 


\title{
Assessing the performance of hospital capacity planning through simulation analysis
}

\author{
Guoxuan Ma \& Erik Demeulemeester \\ Katholieke Universiteit Leuven, Faculty of Business and Economics, Department of Decision \\ Sciences and Information Management \\ Naamsestraat 69, B-3000 Leuven, Belgium \\ Tel: +32-16-32.69.72 \\ Fax: +32-16-32.66.24 \\ guoxuan.ma@econ.kuleuven.be \\ erik.demeulemeester@econ.kuleuven.be
}

\begin{abstract}
Hospital capacity planning at the tactical level produces the optimal patient case mix that can be taken care of at a hospital with the associated time-phased allocation of resources. However, different generated scenarios may result in dissimilar effects on the delivery of health care when variability comes into play. Therefore, it is important to evaluate the performance of capacity planning through simulation analysis. In our setting, the daily booked surgical inpatients are always operated on but possibly are misplaced in an alternative area for recovery because of a bed shortage in the suitable ward, while the bed shortage is the consequence of fluctuating lengths of stay of the patients. Patient misplacement is the chief performance indicator to reflect the service level. Two aspects are studied intensively in this paper. On the one hand, in order to reduce the bed shortage maximally, a few strategies are suggested when determining the capacity decisions. Then, the impacts of different strategies are compared on bed occupancy and patient misplacement. On the other hand, sensitivity analysis is carried out to check whether the number
\end{abstract}


of patient misplacements can be reduced and whether the bed shortage phenomenon can be mitigated.

Keywords: hospital capacity planning, patient misplacement, bed occupancy, variability, discrete-event simulation, sensitivity analysis 


\section{Introduction}

Faced with increasing economic pressures, health service providers (e.g., hospitals) are prone to choose the patient case mix that can bring maximum overall financial contributions under the limited resource capacity and/or that maximizes the resource utilization. Ma et al. (2009) propose a comprehensive methodology to decide the optimal patient mix and the corresponding capacity allocation of multiple resources in a deterministic manner. Basically, aiming at producing the maximum total profits, the elective inpatient cases are selected for admission into a hospital, and simultaneously the operating room (OR) blocks are assigned to each surgeon group and the bed capacity is allocated to various wards to satisfy the demand of the admitted patients. The adopted parameters here, such as the surgery duration and the length of stay (LOS), are all deterministic and are stipulated as the expected value of each patient group. Consequently, an optimal case mix pattern is determined, as well as the time-phased OR blocks assignment and the fixed bed allocation scheme. The selected patient case mix in combination with the cyclic OR blocks assignment will construct a master surgery schedule for the hospital. Additionally, Adan et al. (2009a) incorporate the distributed LOS in their deterministic patient mix model, which aims at maximizing the utilizations of manifold resources when planning the patient case mix in a surgical department, and investigate through a case study the influence of the replacement of the expected LOS by a certain probability distribution.

However, the variability existing in the health care system, such as the random patient arrivals, the variable operating time and the stochastic LOS, will impact the operational performance in the real delivery of the health service and impair the planned utilization of resources. Moreover, the capacity decision on the basis of resource efficiency neglects the patient service level, which may result in disadvantages for the patient treatment and thus in lower patient satisfactions. 
Actually, as Beliën and Demeulemeester (2007) and van Oostrum et al. (2008) indicated, the master surgery schedule affects the resulting daily bed requirement, which may cause the bed shortage in the stochastic environment. Conversely, the bed shortage, because of the fixed bed allocation and the variable LOS of patients, will result in the cancellation of planned surgeries or the misplacement of operated patients, and thus be harmful to the utilization of operating rooms or to the patient treatment. Therefore, it is important for a hospital to determine the selection of the patient mix, the periodical block assignment and the decision on bed capacity in each ward as a whole in the presence of variability.

In fact, the deterministic model proposed in Ma et al. (2009) may generate different scenarios which all contribute the maximum profits to the hospital but perform distinctly in the operational process when the variability comes into play. Hence, it is meaningful to develop a discrete-event simulation model to observe/compare their operational performance and to check whether and how to improve the performance. In our modeling, the hospital is assumed to be configured according to the capacity decision generated from the case mix model, and patients from various groups are admitted on the basis of the constructed master surgery schedule. Supposedly, the scheduled patients are always operated on but may be misplaced in other wards for recovery due to the bed shortage in the suitable ward, which means that there is no surgery cancellation but patient misplacement in our simulated experiments. Patient misplacement means that a patient is moved to a wrong ward to occupy a bed for recovery after his/her surgery performed. Once an occupied bed is released and available in the suitable ward, the patient will be transferred back to continue his/her recovery under more professional care.

Green (2004) points out that the length of the waiting lists in a hospital heavily depends on the capacity structure, the demand side and the variability in the system. Similarly, in our setting the 
patient misplacement is a consequence of three aspects, i.e., the capacity decision, especially the implied master surgery schedule, the variable LOS of each patient group and the size of bed capacity planned. Thus, the performance evaluation will be investigated with regard to the above three factors. The purpose of this paper is two-fold. Firstly, in order to reduce the bed shortage maximally, a few strategies are suggested to balance the daily bed requirements of each ward which are translated into the patient mix model to seek for more reasonable capacity decisions. Then, the various scenarios produced are simulated to compare the operational performance. Secondly, the sensitivity analysis is carried out to check how the hospital's performance is influenced by the variability of the LOS and the volume of beds.

There exists extensive literature on evaluating the performance of hospital capacity planning. Jacobson et al. (2006) provide a literature review on discrete-event simulation of health care systems. Vassilacopoulos (1985) presents a simulation model to determine the number of beds required to meet the hospitalization demand. Butler et al. (1992a) build a simulation model to assess the impact of health care policies on patient misplacement. Butler et al. (1992b) propose an integrative two-phase approach to evaluate bed configurations and to determine optimal bed allocations across a number of hospital service areas. Fitzpatrick et al. (1993) investigate the OR scheduling policies in terms of facility utilization and patient waiting time. Bagust et al. (1999) suggest dynamic use of bed capacity to accommodate emergency patients. Harper and Shahani (2002) highlight the need for evaluating hospital bed capacity in light of both bed occupancies and refused admission rate. VanBerkel and Blake (2007) develop a simulation model to examine the effect of the redistribution of bed capacity on the reduction of patient waits. Adan et al. (2009b) suggest a few practical strategies to improve the operational effectiveness of tactical master surgery plans. In addition, Beliën et al. (2006) develop a software system to visualize the 
impact of the master surgery schedule on the demand for various resources throughout the rest of the hospital. And queueing analysis (Green 2006) is another branch to assess the capacity planning of health care sectors. Gorunescu et al. (2002) and McManus et al. (2004) both apply queueing models to determine the bed capacity accurately.

The remainder of this paper is organized as follows. Section 2 describes the tactical capacity planning in detail. A basic deterministic model is created to decide the optimal case mix and its associated resource allocation scheme, and then the model is modified step by step to potentially advance the patient service levels. In Section 3, a discrete-event simulation model is developed completely through elaborating each component and animating the whole process of patient treatment. Afterwards, a series of capacity decision scenarios derived from the basic patient mix model and its variants are simulated to compare the operational performance by applying an artificial instance in Section 4. Section 5 discusses whether and how to improve the performance through sensitivity analysis. Finally, Section 6 draws conclusions and directs the further steps of this research.

\section{Problem description}

A hospital is assumed to comprise a number of independent units and a common surgical center. Each unit has a few surgeon groups and a private ward with a certain number of beds to admit its patients, and each surgeon group can perform surgeries for several patient groups. Each patient group is characterized uniquely by its consumed resources, such as the average operating time, the mean bed days for recovery and its assigned surgeon group. The surgical center contains a few operating rooms, which are shared by all surgeon groups and are open on workdays only, called active days. An OR block is defined as the smallest time unit for which an operating room can be assigned to a surgeon group exclusively for performing surgeries. 
Basically, the tactical capacity planning problem consists of determining how many OR blocks are assigned to each surgeon group on each active day, how many beds are assigned to each nursing ward and how many patients from each group can be taken care of at a hospital in view of maximizing the overall contribution. As a result, a capacity decision is generated, that is, the fixed allocation of bed capacity, the time-phased assignment of OR blocks as well as the selected patient case mix. The case mix pattern together with the cyclic block assignments determine a master surgery schedule. In order to formulate the capacity planning problem mathematically, a number of needed notations are introduced and defined as follows:

$W$ : set of wards with index $w$;

$S$ : set of surgeon groups with index $s$;

$P$ : set of patient groups with index $p$;

$D$ : set of days in a cycle with index $d$;

$A$ : set of active days in a cycle with index $a$;

$S_{w}$ : subset of surgeon groups $S$ whose patients are moved in ward $w$ after surgery performed;

$P_{w}$ : subset of patient groups $P$ of which the patients occupy the beds of ward $w$;

$P_{S}$ : subset of patient groups $P$ of which the patients are operated on by surgeon group $s$;

$A_{p d}$ : subset of active days on which patients of group $p$ operated still stay in hospital on day $d$;

$r_{p}$ : the profit generated by treating a patient of patient group $p$;

$d u r_{p}$ : the surgery duration time of operating on a patient of patient group $p$;

$L B_{p}$ : the lower bound on the number of patients of group $p$ that can be treated per cycle;

$U B_{p}$ : the upper bound on the number of patients of group $p$ that can be treated per cycle;

$B E D S$ : the total number of available beds (assumed to be the existing bed capacity);

BLOCKS: the total number of available OR blocks (assumed to be the existing block capacity); 
LENGTH: the operating room block length (assumed to be 480 minutes).

And the decision variables include:

$x_{p a}$ : the number of patients of patient group $p$ that receive surgery on day $a$;

$y_{w}$ : the number of beds assigned to ward $w$;

$z_{s a}$ : the number of OR blocks assigned to surgeon group $s$ on day $a$.

Then, an integer linear programming (ILP) model is formulated as below:

$$
\begin{aligned}
\max & \sum_{p \in P} r_{p} \sum_{a \in A} x_{p a} \\
\text { s.t. } & \sum_{w \in W} y_{w} \leq B E D S, \\
& \sum_{p \in P_{w}} \sum_{a \in A_{p d}} x_{p a} \leq y_{w}, \quad \forall w \in W, d \in D, \\
& \sum_{s \in S} z_{s a} \leq B L O C K S, \quad \forall a \in A, \\
& \sum_{p \in P_{s}} d u r_{p} x_{p a} \leq z_{s a} L E N G T H, \quad \forall s \in S, a \in A, \\
& L B_{p} \leq \sum_{a \in A} x_{p a} \leq U B_{p}, \quad \forall p \in P, \\
& x_{p a}, y_{w}, z_{s a} \in \mathrm{N}, \quad \forall w \in W, s \in S, p \in P, a \in A .
\end{aligned}
$$

The objective function (1) aims at maximizing the total contributions. Constraint (2) indicates the allocation of bed capacity. Constraint set (3) denotes that the daily bed occupancy of each ward cannot exceed its allocated capacity. Constraint set (4) reflects the time-phased assignment of OR blocks. Constraint set (5) indicates that the total surgery time for a surgeon group on an active day cannot exceed the assigned block length. Also the patient admission volume constraint (6) and the integer variable constraint (7) are listed. 
The above ILP model is a huge integer program even for a hospital of normal size (e.g., 20 units), which means it may generate many different capacity decisions contributing the maximum total profits but behaving distinctly in the operational process when variability comes into play. Therefore, in order to both optimize the resource efficiency and advance the patient service, three strategies are suggested and formulated in the following. They are (1) averaging the possible spare beds, (2) replacing the expected LOSs by the distributed ones, and (3) adopting the flexible block length respectively.

\section{1. $\quad$ Averaging the spare beds}

Firstly, according to the numerical experiments of the ILP model (1)-(7), the possible spare beds are inclined to being gathered in one or a small number of wards. Then, after taking into account the variable LOS in reality, the fluctuating daily bed demands at most wards will result in a large bed shortage. Hence, in order to mitigate or reduce the bed shortage of most wards, averaging the possible spare beds will be feasible and effective.

A new variable $t_{w d}$ is introduced to denote the number of unoccupied beds at ward $w$ on day $d$. Then, constraint (3) is modified into the below form:

$$
\sum_{p \in P_{w}} \sum_{a \in A_{p d}} x_{p a}+t_{w d}=y_{w}, \quad \forall w \in W, d \in D .
$$

In order to reduce the potential bed shortage, each ward is expected to possess a flat distribution of daily bed occupancy and a certain number of spare beds. Thereby, we define a minimum volume of spare beds for all ward days, and aim to maximize the minimum value. Consequently, the objective function is changed as

$$
O B J=\alpha * \sum_{p \in P} r_{p} \sum_{a \in A} x_{p a}+\beta * \min S B,
$$

where 


$$
\min S B=\min _{\forall w \in W, d \in D}\left\{t_{w d}\right\}, \quad \alpha \gg \beta
$$

The objective function implies that the possible spare beds are averaged over each ward on the condition that the maximum total contribution is achieved at first. Thus, the assigned spare beds may compensate the bed shortage because of the fluctuating daily bed demands.

\subsection{Replacing the expected LOS by its distribution}

Secondly, in order to calculate the daily bed requirement of each ward more accurately, the expected LOS of each patient group is replaced by its distribution in the basic model. Actually, Adan et al. (2009a) definitely illustrate that applying the stochastic LOS in place of its mean value will result in a better patient admission profile in terms of resource utilizations. Utley et al. (2005) clearly explain that by using the probability distribution of the LOS the expected bed occupancy of ward $w$ on day $d$ can be computed as

$$
\mu_{w, d}=\sum_{p \in P_{w}} \sum_{a \in A} x_{p a} \sum_{k \geq 0} \operatorname{prob}_{p, k T+d-a}
$$

in which $\operatorname{prob}_{p, l}(l \geq 0)$ refers to the probability that a patient of group $p$ is still an inpatient after $l$ days of his/her surgery performed. Now, constraint (3) is rewritten as

$$
\mu_{w, d}+t_{w d}=y_{w}, \quad \forall w \in W, d \in D .
$$

Here, it should be pointed out that the expected bed occupancy $\mu_{w, d}$ is not necessarily an integer because of the real probability $\operatorname{prob}_{p, l}$. Thus, the same applies to the volume of spare beds $t_{w d}$. Moreover, $t_{w d}$ can be negative since the expected bed requirement solved from the distributed LOS may exceed the assigned bed capacity. Then, $t_{w d}$ means the expected bed shortage of ward $w$ on day $d$ but not the volume of spare beds, and the minimum negative value minS $B$ reflects the maximum bed shortage for all ward days. Thus, the objective function (8) now implies either maximizing the minimal volume of spare beds $(\min S B>0)$ or minimizing the maximal expected bed shortage $(\min S B<0)$ on the basis of achieving the maximum overall rewards. 


\subsection{Adopting a flexible block length}

Thirdly, as Beliën and Demeulemeester (2007) and van Oostrum et al. (2008) indicated, master surgery schedule affects the resulting bed demand. Therefore, in order to balance the daily bed occupancy of each ward maximally, a flexible block length is suggested to produce the master surgery schedule with a more flat distribution of bed demands over a cycle. Here, two strategies are applied to implement a flexible block length. One strategy consists of dividing each one day OR block into two sessions, i.e., a morning session and an afternoon session. Another strategy consists of permitting overtime for the operating rooms systematically, for example extending each OR block by one hour. These techniques can be introduced into the ILP model through changing the constraint (5) as

$$
\sum_{p \in P_{s}} d u r_{p} x_{p a} \leq \rho \cdot z_{s a} L E N G T H, \quad \forall s \in S, a \in A,
$$

where the parameter $\rho>1$ (e.g., $\rho=1.2$ ) indicates overtime of the OR blocks or $\rho=0.5$ means dividing each block in half.

After adopting the flexible surgery blocks, the patient admission maybe increases due to more OR capacity provided. However, more patient admission implies more bed days demand. Thus, in order to compare the bed occupancy of different capacity decisions, the patient admission standard is kept the same as the one solved from the basic model, that is,

$$
\sum_{a \in A} x_{p a}=T H R_{p}, \quad \forall p \in P
$$

where $T H R_{p}$ denotes the solved patient throughput of group $p$ within a cycle.

In addition, it is possible to integrate both strategies of flexible surgery blocks to produce a hybrid method. For example, dividing an 8 hour OR block into one session from 8am to 12am and another session from 2pm to 6pm, and then overrunning each session for 45 minutes. 
Accordingly, through adding the above strategies incrementally to the basic capacity planning problem, six categories of capacity decisions are generated. They are called respectively as the base mode solved from the basic model, the balanced mode after averaging the possible spare beds, the distributed mode by utilizing the stochastic LOS, also the overtime mode, the 2-session mode and the hybrid mode when adopting the flexible surgery blocks.

\section{Simulation modeling}

In this section, a discrete-event simulation model is developed through applying the user-friendly simulation software Arena version 11.0 (Kelton et al. 2008). The simulation model consists of three main logical parts, that is, the patient arrival suite, the surgical center suite and the ward suite. In the following, each suite is described in detail, also the data modules are explained clearly. After that, the model verification and validation are implemented through investigating and animating an example of a hospital with two departments.

\subsection{A generic simulation model}

Firstly, a generic simulation model is presented. An elective patient with a certain pathology enters into a department of a hospital according to his/her own timing. After the diagnosis, the patient is classified into a patient group and put on a corresponding waiting list. Normally, the patient waits at home for an available operating block to undergo surgery. When the block is accessible, the patient is moved to an operating room and is operated on by a certain surgeon group. After that, he/she will be transferred into a suitable ward and occupy a bed to recover. The occupied bed will be allocated to other patients after the patient finishes his/her recovery in the ward. If there is no free bed available at the most suitable ward, the operated patient will have to be misplaced in an another ward or on a temporary bed, until a suitable bed is set free and available. Figure 1 depicts the basic structure of the patient treatment process in the hospital. 


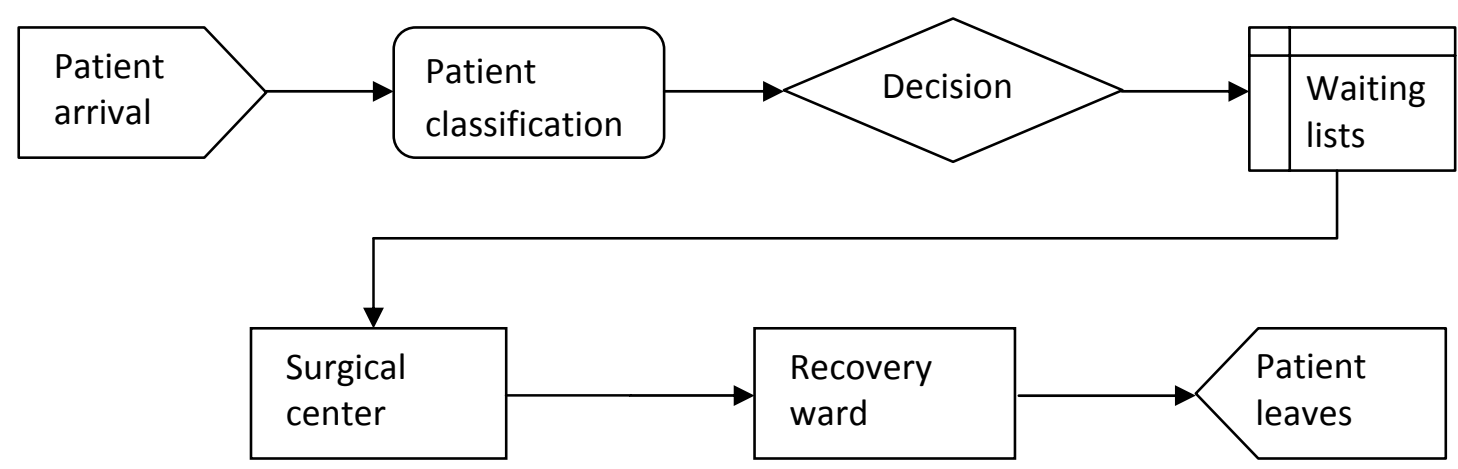

Figure 1: Basic structure of the model

\subsection{Patient arrival suite}

Patients arrive at a hospital randomly based on their own timing, and their arrivals follow a nonstationary Poisson process for each patient group in general. The process of the patient arrival is implemented through building a create module and specifying a detailed probability distribution for the arrivals of each group. Since the patient admission is based on the selected case mix, and since the performance indicator on service level is the patient misplacement but not the patient waiting time, it is assumed that the volume of patient arrivals of each group is in accordance with the solved case mix pattern. If the patient arrivals differ from the case mix, patient throughputs will be below the planned ones and/or long waiting lists will be incurred.

Patients with a certain pathology enter into one department of the hospital, directed by the general practitioner or their family doctors. Then, they are classified into different patient groups based on their diagnosis results. Each patient group has a waiting queue, which follows the principle of first-come-first-serve. Before the surgery is performed, each patient has to wait in the belonged queue. Since all patients considered are not urgent but elective, there is no priority rule for the waiting lists. Finally, it is assumed that no registered patient leaves the queue automatically before their surgeries are performed and no scheduled surgery is cancelled because of other reasons (e.g., bed shortage) in the whole process. 


\subsection{Surgical center suite}

The surgical center has a fixed number of operating rooms, which are shared and utilized by all surgeon groups to perform surgeries only for elective patients. The operating rooms are assigned to various surgeon groups in a timed fashion. Once a room is assigned to a surgeon group on a block, it will be utilized exclusively by the assigned surgeon group to operate on its planned patients. If there are not enough planned patients in the corresponding waiting queue, normally the operating room will not be utilized completely. Actually, scheduling other unplanned patients can improve the OR utilizations, but simultaneously generates different bed demands in the ward and definitely affects the bed occupancy and the patient service. Therefore, in our experiments we assume that unplanned patients cannot be operated on. If the scheduled surgeries run out of the assigned blocks due to the variable surgery time, overtime is allowed to finish the scheduled surgeries. Overtime to some extent is common in most hospitals and therefore it is accepted somehow. Moreover, overtime can avoid the cancellation of the scheduled surgeries.

Since the purpose of the simulation analysis is mainly focused on observing and relieving the bed shortage phenomenon, the stochastic operating time, which only affects the OR utilizations, is simplified here. Actually, the operating time of each patient is assumed to be constant and equal to the expected surgery duration of its patient group. In addition, each block is assumed to be 8 hours, but may be extended in the case of flexible surgery blocks. Operating rooms are only open from Monday to Friday, but closed in the weekend. The surgeon groups are assumed to be always available during their assigned OR blocks.

\subsection{Ward suite}

Each department is assumed to own a personal ward with a number of beds for hospitalizing its patients. And the bed capacity is divided over various wards according to the achieved capacity 
decision in the deterministic step. Besides the regular beds allocated to each nursing ward, a few temporary beds are assumed to be located at a common area in the hospital, called a "shadow ward" for instance. In the real configuration of a hospital, temporary beds may be dispersed in the existing wards, such as the unstaffed backup beds. Supposedly, hospital beds in each ward accommodate patients from its correlated department, but they are also allowed to hospitalize patients from other adjacent departments shortly when there is no free bed available in their wards. And, when all regular beds are occupied in the whole hospital, a number of temporary beds is defined to put the remaining patients temporarily. If an operated patient is moved to a second suitable ward for recovery, it is called patient misplacement. The misplaced patient will be reassigned to the most suitable ward once there are free beds available. The length of a patient misplacement is called the patient misplaced bed days (MBDs).

The LOS of each patient is generated randomly from a certain distribution of the LOS of its patient group. If a patient is transferred to the suitable ward, he/she will occupy a bed for his/her LOS continuously and release the bed after getting recovered and leaving the hospital. For the misplaced patients, it is assumed that the misplacement does not affect the patients' recovery and extend their LOSs. After being transferred to the proper ward, the patient will continue the recovery until finishing his/her stays.

\subsection{Data modules}

In order to concretize the simulation model, some necessary data should be specified in detail. In our model, the needed data not only consist of the probability distribution of the patient arrivals of each group, the time-phased OR blocks assignment and the bed capacity allocation to each ward, but also comprise the constant surgery duration and the distribution of the LOS of each patient group. All the data applied in the simulation model are in accordance with the solution 
and parameters of the patient mix model. In order to run the simulation model correctly, the data will have to be specified accordingly by using the data modules of Arena.

\subsection{Verification and validation}

Every simulation model should be thoroughly verified and validated in order to achieve valuable results. The verification process, on the one hand, is to ensure that the simulation model behaves in the way as it was intended according to the modeling assumptions made, and it is situated in the transition from the conceptual model to the computerized representation. The validation process, on the other hand, is to guarantee that the model behaves the same as the real system that will be examined, and it is located in the transition from the real system to the simplified conceptual model. Although the goal of both processes is different, they are mostly conducted simultaneously.

We apply two basic approaches to verify the simulation model. Firstly, we continuously check whether the outputs of the simulation runs are reasonable. This is done both for regular runs and the special runs under extreme conditions. For example, we allow for only one patient to enter the system and follow the patient to examine whether the model logic and data are correct; we make the long experiment runs for different data and observe the summary results for potential problems; we replace some or all the variability with constants and run the simulation model to check the system behavior and the results exactly. Secondly, we develop a structured and vividly animated graphical representation for our simulated model. The animation could help to identify the regularly running manner and any abnormalities of the system. Figure 2 provides a snapshot of this graphical representation.

During the conduct of the example experiment, we especially pay attention to the determination of the warm-up period and the number of replications in order to get reliable outcomes. 


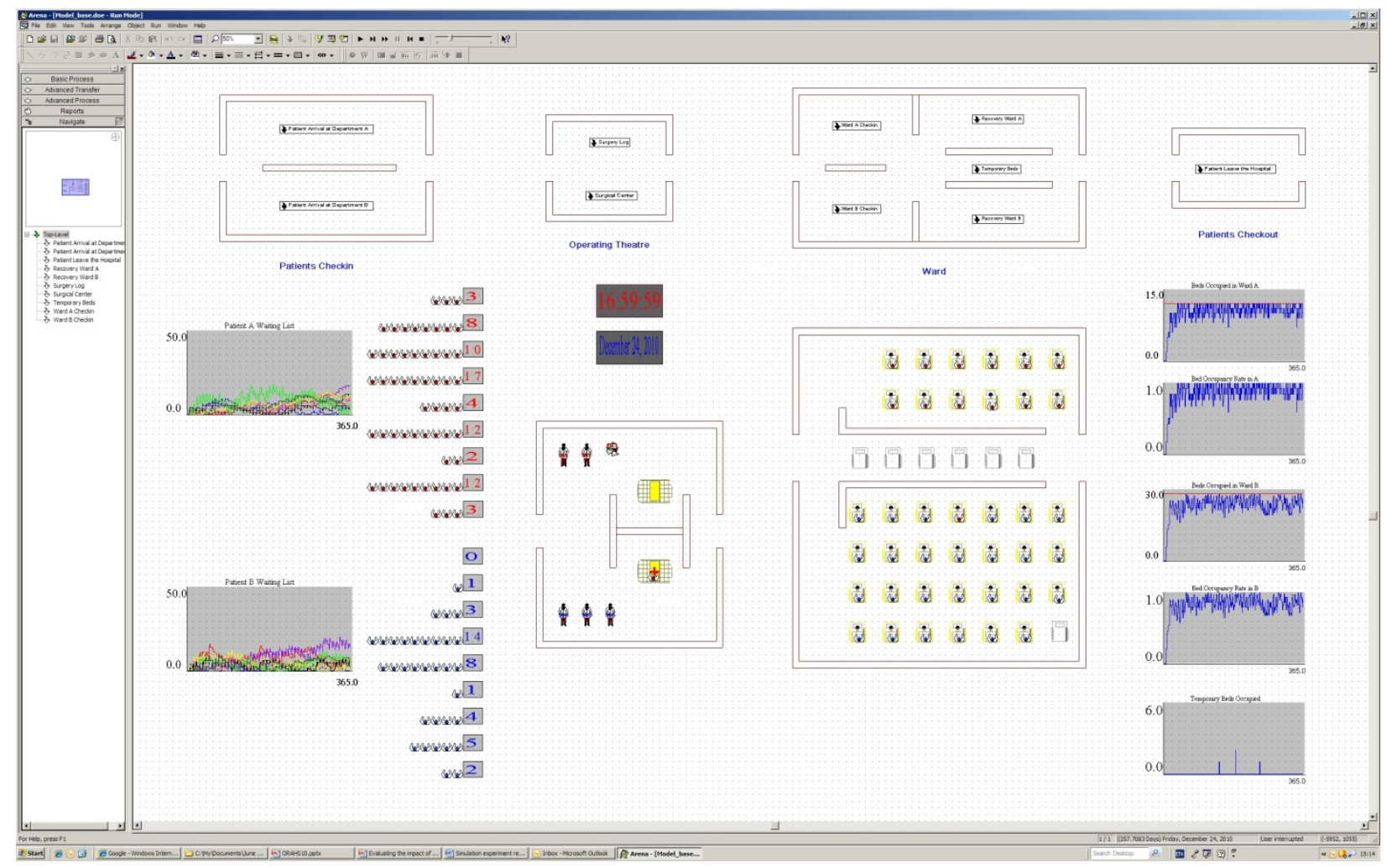

Figure 2: Graphical representation and animation of the model

\section{Comparison results}

In this section, we illustrate the impact of capacity decisions on hospital performance through an example of a two-unit hospital. Capacity decisions generated under different policies mentioned before are simulated in the constructed model in order to observe their operational performances. The experimental results are compared through a series of performance indicators, which consist of the volume of patients misplaced, the patient misplaced bed days, the bed occupancy of each ward and the utilization of temporary beds.

The two-unit hospital is assumed to comprise 2 operating rooms and 40 hospital beds in total. Each department has three surgeon groups, and each surgeon group can perform surgeries for three patient groups. Data on the expected surgery duration, the average LOS and reward of each patient group are generated randomly and the distribution of the LOS for each patient group is 
assumed reasonably. Besides the associated two wards (Ward A and Ward B respectively), six temporary beds are assumed to be located in area $\mathrm{T}$. The basic model produces a bed allocation scheme: 12 beds assigned to ward A and 28 beds assigned to B. However, in other situations 13 beds are assigned to ward A and 27 beds are assigned to ward B. Table 1 displays the compared simulation results of six capacity decisions generated under different policies.

\begin{tabular}{|c|c|c|c|c|c|c|c|}
\hline \multicolumn{2}{|c|}{ Indicators } & Base & Balanced & Distributed & Overtime & 2-session & Hybrid \\
\hline \multirow{4}{*}{$\begin{array}{c}\text { Patient } \\
\text { misplaced }\end{array}$} & $\mathrm{A}$ in $\mathrm{T}$ & 6.20 & 3.78 & 3.30 & 1.92 & 0.26 & 0.80 \\
\hline & $A$ in $B$ & 25.18 & 5.12 & 6.00 & 4.04 & 8.18 & 4.88 \\
\hline & A's MBD & 31.38 & 8.90 & 9.30 & 5.96 & 8.44 & 5.68 \\
\hline & $\mathrm{B}$ in $\mathrm{T}$ & 2.44 & 2.02 & 1.58 & 1.30 & 2.80 & 2.60 \\
\hline \multirow{4}{*}{$\begin{array}{l}\text { bed days } \\
\text { (MBD) }\end{array}$} & $B$ in $A$ & 6.96 & 12.02 & 12.16 & 16.78 & 11.16 & 12.08 \\
\hline & B's MBD & 9.40 & 14.04 & 13.74 & 18.08 & 13.96 & 14.68 \\
\hline & $\mathrm{AB}$ in $\mathrm{T}$ & 8.64 & 5.80 & 4.88 & 3.22 & 3.06 & 3.40 \\
\hline & Total & 40.78 & 22.94 & 23.04 & 24.04 & 22.40 & 20.36 \\
\hline \multirow{3}{*}{$\begin{array}{c}\text { Misplaced } \\
\text { patients }\end{array}$} & $\mathrm{A}$ in $\mathrm{T}$ & 5.70 & 3.46 & 3.04 & 1.66 & 0.26 & 0.80 \\
\hline & $A$ in $B$ & 23.70 & 4.94 & 5.84 & 4.04 & 8.14 & 4.86 \\
\hline & $\mathrm{B}$ in $\mathrm{T}$ & 2.32 & 1.96 & 1.52 & 1.28 & 2.74 & 2.60 \\
\hline volume & $\mathrm{B}$ in $\mathrm{A}$ & 6.80 & 11.92 & 11.94 & 16.72 & 11.04 & 12.02 \\
\hline \multirow{2}{*}{$\begin{array}{c}\text { Bed } \\
\text { occupancy }\end{array}$} & Ward A & 0.8581 & 0.7933 & 0.8010 & 0.7976 & 0.8001 & 0.8027 \\
\hline & Ward B & 0.8494 & 0.8732 & 0.8733 & 0.8746 & 0.8753 & 0.8739 \\
\hline \multirow{4}{*}{$\begin{array}{l}\text { Temporary } \\
\text { beds } \\
\text { utilization }\end{array}$} & Bed 1 & 1.3797 & 0.8771 & 0.7051 & 0.5483 & 0.4205 & 0.5557 \\
\hline & Bed 2 & 0.3991 & 0.3218 & 0.2339 & 0.1336 & 0.1506 & 0.1208 \\
\hline & Bed 3 & 0.1295 & 0.0580 & 0.0951 & 0.0362 & 0.0405 & 0.0271 \\
\hline & Bed 4 & 0.0087 & 0.0129 & 0.0386 & 0 & 0.0038 & 0.0197 \\
\hline (\%) & Bed 5 & 0.0043 & 0.0043 & 0.0043 & 0 & 0 & 0 \\
\hline
\end{tabular}

Table 1: Comparison results of different scenarios 
In Table 1, patient misplacements are divided into two classes: misplaced in the neighbor ward and misplaced in area T. For example, if a patient from department A is misplaced in ward B, it is recorded as "A in B", and if patient $\mathrm{A}$ is misplaced in area $\mathrm{T}$, we record it as "A in T". From the table, we can see that the patient misplacement is reduced and the utilization of temporary beds is declined on the whole, after taking the proposed strategies into account when solving the capacity decisions. We also observe that the patient misplacements of unit B in Balanced mode are higher than the ones in Base mode, which attributes to the bed capacity allocation to ward B changed from 28 to 27 after averaging the possible spare beds. However, patient misplacements of department A and total patient misplacements both are decreased, which correctly reflects that the strategy of averaging spare beds is valid to improve the service level.

From Table 1, we also observe that the total patient misplaced bed days have slight increments from the balanced mode (22.94) to the distributed mode (23.04) then to the overtime mode (24.04). However, the patient misplaced bed days in temporary beds are decreased distinctly, that is, from 5.80 of the balanced mode to 4.88 of the distributed mode then to 3.22 of the overtime mode, which clearly demonstrates that applying the suggested strategies incrementally could effectively reduce the patients being misplaced in temporary beds and the bed shortage in the whole hospital. Actually, this is also reflected by other performance indicators. The volume of misplaced patients $\mathrm{A}$ in temporary beds is declined from 3.46 to 3.04 then to 1.66 , and the volume of misplaced patients B in temporary beds is declined from 1.96 to 1.52 then to 1.28. As well as the temporary bed utilization is reduced evidently. In addition, compared to the overtime mode, the 2-session mode has better performance results on the whole except for the bed days of patients B misplaced in temporary beds. Finally, we can see that the hybrid mode, which combines all the strategies, has the smallest total patient misplacements. 
Therefore, we can conclude that the proposed policies are effective and valuable to balance the bed occupancy of each ward and then to reduce the bed shortages.

\section{Sensitivity analysis}

In this section, the sensitivity analysis is carried out to check whether the number of patient misplacements can be reduced and how the bed shortage phenomenon can be mitigated. Two approaches are suggested and executed in the simulation experiments. They are (1) curtailing the distribution of the LOS of some patient groups (from the perspective of variability) and (2) expanding the bed capacity appropriately (from the perspective of capacity) respectively. The trade-offs between resource efficiency and patient service are analyzed, in which the resource efficiency is reflected by the bed occupancy rate of each ward and the service level is expressed by the number of observed patient misplacements.

The variable LOS of patients impacts the bed occupancy, which may result in a shortage of beds in each ward temporarily. This motivates us to check the effects of the varied LOS on the patient service level. Curtailing the distribution of the LOS of one patient group means cutting the tail of the distribution and reshaping it in order to keep the mean value of the LOS being constant. It possibly happens when the surgery procedure is improved or the nursing program is changed. On the average, the total demand for bed days is not altered, but the daily bed requirement of each ward may be varied. Table 2 lists partial results of the simulation experiments for the capacity decisions of the base mode and the balanced mode.

Clearly, after curtailing the distribution of the LOS of some patient groups, not only the total patient misplacements of each department are reduced evidently, but also the total misplaced bed days in temporary beds are decreased a lot. Moreover, from Table 2 we also see that the bed occupancy rate of each ward is slightly changed, which correctly reflects that the higher the 
degree of variability in the system, the worse the patient service under the same utilization level (Green 2006). And, the slight increment of bed occupancy is in concert with the reduction of patients being misplaced in temporary beds, which are changed to occupy a regular bed instead.

\begin{tabular}{|c|c|c|c|c|c|}
\hline \multicolumn{2}{|c|}{ Indicators } & Base & Curtailed & Balanced & Curtailed \\
\hline \multirow{3}{*}{$\begin{array}{c}\text { Misplaced } \\
\text { bed days }\end{array}$} & A's MBD & 31.38 & 26.86 & 8.90 & 6.28 \\
\cline { 2 - 6 } & B's MBD & 9.40 & 4.88 & 14.04 & 9.26 \\
\cline { 2 - 6 } & AB in T & 8.64 & 4.54 & 5.80 & 2.52 \\
\hline \multirow{3}{*}{$\begin{array}{c}\text { Bed occupancy rate } \\
\text { \& Occupied beds }\end{array}$} & Ward A & 0.8581 & 0.8592 & 0.7933 & 0.7929 \\
\cline { 2 - 6 } & Ward B & 10.2966 & 10.3106 & 10.3129 & 10.3083 \\
\cline { 2 - 6 } & & 23.7827 & 23.7822 & 23.5759 & 23.5933 \\
\hline
\end{tabular}

Table 2: Results of curtailing the LOS

Besides variability, the size of capacity with its utilization is another factor affecting the patient service. Green (2006) indicates that historically an average bed occupancy rate of 85 percent has been used as the minimum level for the hospital to make a decision on bed expansion. In our experiments, the results show that the average hospital bed occupancy is close to the minimum level. Hence, it is interesting to check what happens if the bed capacity is changed somehow. In our example, the generated patient case mix needs at least 38 beds to satisfy their demand for recovery in the deterministic condition, and the bed allocation scheme is 12 beds assigned to ward A and 26 beds assigned to ward B. Thus, we simulate the bed capacity decision from the minimum level (38 beds) and progressively expand the volume of beds to a corresponding ward. The simulation results are displayed in Figure 3. At the minimum level, ward B has a high bed utilization (>90\%) which implies that more beds are needed, and the patient misplacement status is terrible. After introducing one bed to ward B, its bed occupancy rate is decreased considerably, 
also the bed utilization of ward A is reduced a little. Simultaneously, the patient misplaced bed days decline sharply. When the size of bed capacity is increased to 41 (A13vsB28), both wards have an occupancy rate less than the $85 \%$ baseline and the patient misplacement is ameliorated a lot, especially the patient misplaced bed days in temporary beds coming close to zero.

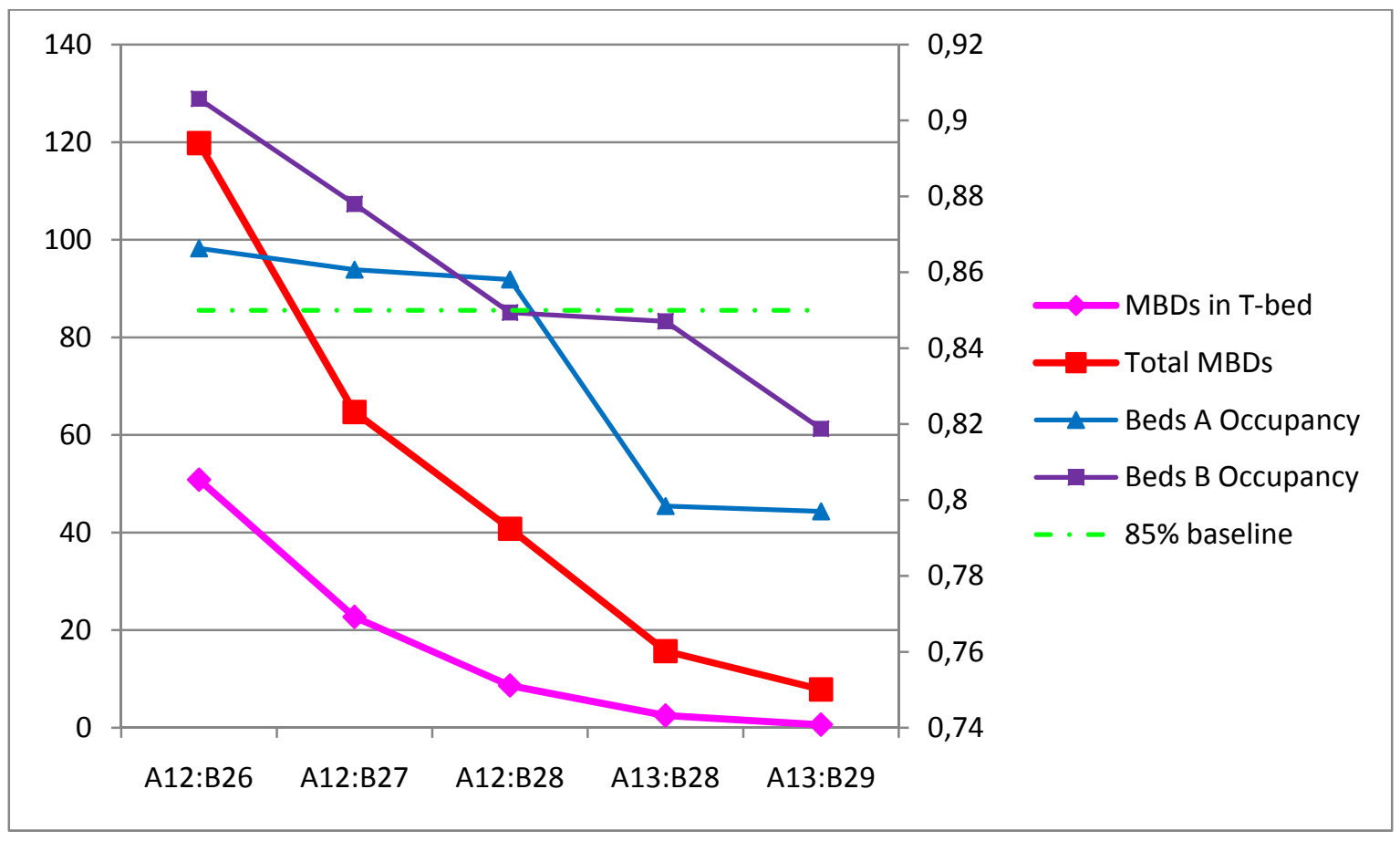

Figure 3: Trade-offs between bed occupancy and patient misplacement

\section{Conclusion and future work}

In this research, the operational performance of a generated hospital capacity decision is assessed through using discrete-event simulation. The capacity decision is based on a deterministic mathematical model, which cannot truly reflect the impact of variability existing in hospitals. Therefore, it is of necessity to simulate the hospital's capacity planning, taking into account the stochastic property in the health care delivery.

Simulation experiments clearly indicate that the effects of different capacity decisions on patient service level are distinct. And a 'better' capacity scenario with the improved performance can be sought after taking into account more reasonable conditions in the decision model. We propose 
three strategies to decrease the bed shortage and improve the patient service, and the experiment results show that they are effective to reduce the patient misplacement. In addition, it is shown that both the distribution of the LOS and the size of bed capacity distinctly affect the hospital's occupancy and patient service, which clearly illustrates that the patient misplacement can be reduced and the bed shortage phenomenon can be mitigated.

The observed patient service is mainly discussed in terms of patient misplacement. However, the patient waiting length or surgery cancellations are not considered in this paper. Hence, it may be interesting to take the surgery cancellation into account and to study its effect on patient delays. In addition, the variable surgery durations may result in overtime in operating rooms and the fluctuating patient arrivals may damage the effect of capacity planning, for example the intended patient throughput cannot be achieved, and so on.

\section{Acknowledgements}

We acknowledge the support given to this project by the Fonds voor Wetenschappelijk Onderzoek (FWO)-Vlaanderen, Belgium under contract number G.0456.08.

\section{References}

Adan I, Bekkers J, Dellaert N, Vissers J, Yu X (2009a) Patient mix optimisation and stochastic resource requirements: A case study in cardiothoracic surgery planning. Health care management science 12(2): 129-141.

Adan I, Bekkers J, Dellaert N, Jeunet J, Vissers J (2009b) Improving operational effectiveness of tactical master plans for emergency and elective patients under stochastic demand and capacitated resources. Eurandom Report (Ext. rep. 2009-052). Eindhoven: Eurandom.

Bagust A, Place M, Posnett JW (1999) Dynamics of bed use in accommodating emergency admissions: Stochastic simulation model. British Medical Journal 319: 155-158. 
Beliën J, Demeulemeester E (2007) Building cyclic master surgery schedules with leveled resulting bed occupancy. European Journal of Operational Research 176(2):1185-1204.

Beliën J, Demeulemeester E, Cardoen B (2006) Visualizing the demand for various resources as a function of the master surgery schedule: A case study. Journal of Medical Systems 30(5): 343350.

Butler TW, Reeves GR, Karwan KR, Sweigart JR (1992a) Assessing the impact of patient care policies using simulation analysis. Journal of the Society for Health Systems 3(3): 38-53.

Butler TW, Karwan KR, Sweigart JR, Reeves GR (1992b) An integrative model-based approach to hospital layout. IIE Transactions 24(2): 144-152.

Fitzpatrick KE, Baker JR, Dave DS (1993) An application of computer simulation to improve scheduling of hospital operating room facilities in the United States. International Journal of Computer Applications in Technology 6(4): 215-224.

Gorunescu F, McClean SI, Millard PH (2002) A queuing model for bed-occupancy management and planning of hospitals. Journal of the Operational Research Society 53(1): 19-24.

Green L (2004) Capacity planning and management in hospitals. In: Brandeau ML, Sainfort F, Pierskalla W (ed) Operations research and health care: A handbook of methods and applications. Kluwer Academic Publishers, Boston, pp 15-41.

Green L (2006) Queueing analysis in healthcare. In: Hall RW (ed) Patient flow: Reducing delay in healthcare delivery. Springer Science, New York, pp 281-308.

Harper PR, Shahani AK (2002) Modelling for the planning and management of bed capacities in hospitals. Journal of the Operational Research Society 53(1): 11-18.

Jacobson SH, Hall SN, Swisher JR (2006) Discrete-event simulation of health care systems. In: Hall RW (ed) Patient flow: Reducing delay in healthcare delivery. Springer Science, pp 211-252. 
Kelton WD, Sadowski RP, Sturrock DT (2008) Simulation with Arena(Fourth Edition). McGraw Hall, New York.

Ma G, Beliën J, Demeulemeester E, Wang L (2009) Solving the strategic case mix problem optimally by using branch-and-price algorithms. Proceedings of the 35th International Conference on Operational Research Applied to Health Services. Leuven, Belgium.

McManus ML, Long MC, Cooper A, Litvak E (2004) Queuing theory accurately models the need for critical care resources. Anesthesiology 100(5): 1271-1276.

Utley M, Gallivan S, Jit M (2005) How to take variability into account when planning the capacity for a new hospital unit. In: Vissers J, Beech R (ed) Health Operations Management: Patient flow logistics in health care. Routledge, New York, pp 146-161.

VanBerkel P, Blake J (2007) A comprehensive simulation for wait time reduction and capacity planning applied in general surgery. Health Care Management Science 10(4): 373-385.

van Oostrum JM, Van Houdenhoven M, Hurink JL, Hans EW, Wullink G, Kazemier G (2008) A master surgical scheduling approach for cyclic scheduling in operating room departments. OR Spectrum 30(2): 355-374.

Vassilacopoulos G (1985) A simulation model for bed allocation to hospital inpatient departments. Simulation 45(5): 233-241. 